\title{
Measurement of the charge asymmetry in top quark pair production at the Tevatron
}

\author{
Regina Demina ${ }^{* \dagger}$ \\ University of Rochester \\ E-mail: regina@pas.rochester.edu
}

Recent results on the asymmetries in top-antitop pair production obtained by the Tevatron collaborations are reviewed. In addition to measuring fully reconstructed forward-backward asymmetries the experiments produced results using Legendre polynomial expansion, asymmetry in lepton direction from top-antitop decay and studies of its kinematic dependencies.

The European Physical Society Conference on High Energy Physics -EPS-HEP2013

18-24 July 2013

Stockholm, Sweden

\footnotetext{
* Speaker.

${ }^{\dagger}$ For CDF and D0 collaborations
} 


\section{Introduction}

Top quark discovered by the Tevatron in 1995 [1] is the heaviest of all known particles. The strength of top quark coupling to the newly discovered Higgs boson [2] associated with the spontaneous breaking of the electroweak symmetry is very close to unity. This fact led people to think that top quark may play a special role in this phase transition [3]. In the process of electroweak symmetry breaking gauge bosons that have different couplings to left- and right-handed fermions acquire mass, while the linear combination that has equal couplings remains massless. Some models suggest that a similar mechanism works in the strong sector, giving rise to heavy mediators of strong interaction that have axial component, so called axigluons [4]. Top pair production in $p \bar{p}$ collisions mediated by axigluons would result in parity violation, experimentally observable as a forward-backward asymmetry, not unlike forward-backward asymmetry in fermion production in $e^{+} e^{-}$collisions mediated by the Z-boson. Different classes of models also suggest non-zero forward-backward asymmetry in top pair production [5].

In the Standard Model the asymmetry appears at the $\alpha_{s}^{3}$ level. The interference between Born and box diagrams of $t \bar{t}$ production results in a final state without extra patrons and yields positive asymmetry, while the interference between diagrams containing initial or final state radiation results in negative asymmetry. The overall effect is positive though numeric predictions range from $4 \%$ to $9 \%[6]$.

We define the positive direction to be that of proton, while the negative to be that of antiproton. The asymmetry is evaluated based on the difference in rapidity of top quark with that of anti top quark $(\Delta y)$, a variable that is invariant under the boost along the beam axis. The forwardbackward asymmetry $\left(A_{\mathrm{FB}}\right)$ is the difference between the number of $t \bar{t}$ events with positive and the ones with negative $\Delta y$ divided by their sum. Similarly, the asymmetry in lepton direction can be defined based on the signed rapidity $\left(q_{l} y_{l}\right)$ of the lepton from $t \bar{t}$ decay $\left(A_{\mathrm{FB}}^{l}\right)$. Experimentally, measurement of the lepton-based asymmetry is more robust. Theoretically, the measurement of this observable and a study of its kinematic dependencies provide an additional discriminator between different models [7]. Top pair production signatures are classified based on the $W$ boson decay mode, resulting in three classes of events: dileptons, where both $W$ bosons decayed leptonicaly, $l+$ jets, where only one $W$-boson decays leptonically, and all-hadronic, with both $W$ bosons decaying hadronically. Only channels containing leptons are suitable for the asymmetry measurement, which requires determination of the charge of the top quark. Dilepton channel, though lower in statistics than $l+$ jets channel, offers an additional possibility to measure the asymmetry based on the difference in rapidity between the positive and negative leptons $\left(A_{\mathrm{FB}}^{l l}\right)$.

The first measurement of the $t \bar{t}$ production asymmetry was produced by D0 in 2008 [8], followed by CDF [9]. Despite large statistical uncertainties it was evident that both results based on approximately $1 \mathrm{fb}^{-1}$ of data were considerably higher that any SM prediction that existed at that moment [10]. Updated results from both experiments based on about $5 \mathrm{fb}^{-1}$ of data still exceeded the SM prediction $[11,12]$. In addition, CDF observed a strong dependence of the asymmetry on the invariant mass of the $t \bar{t}$ system $\left(m_{t \bar{t}}\right)$, while D0 reported a large asymmetry in the production of leptons from $t \bar{t}$ decay. $m_{t \bar{t}}$ dependence observed by D0 was in statistical agreement with both the CDF measurement and the SM prediction, similar statement is true for the $A_{\mathrm{FB}}^{l}$ observed by CDF. The apparent deviations prompted model building beyond the standard model (BSM) that 
would explain the observed effect. At about the same time LHC started its operation. While the $t \bar{t}$ charge asymmetry measurement is substantially more difficult and only with the full statistics of the 7 and $8 \mathrm{TeV}$ runs it starts challenging the Tevatron results, LHC reach in search of other new phenomena is far superior to that of the Tevatron. No deviations from the SM were found by the LHC significantly limiting the class of models that could explain the observed $A_{\mathrm{FB}}$. Meanwhile, the SM based calculations of the effect became more sophisticated predicting a number that exceeded the original estimates by a factor 2-3 [13]. Yet, the full evaluation of the effect at the $\alpha_{s}^{4}$ level is still pending [14].

With the Tevatron demise in 2011 the emphasis in its data analysis shifted towards maximizing the statistical sensitivity of the available dataset. To achieve this goal CDF performed the Legendre polynomial expansion analysis, while D0 extended the leptonic asymmetry to the $l+3$ jets sample and performed its kinematic dependence studies. In the dilepton channel D0 measured both $A_{\mathrm{FB}}^{l}$ and $A_{\mathrm{FB}}^{l l}$.

\section{Legendre polynomial expansion of the $t \bar{t}$ production cross section}

The classical definition of the observable as a forward-backward asymmetry is motivated largely by analogy with the property observed in interactions mediated by Z-boson. At the same time this measurement does not not fully exploit the information from the shape of the angular distribution. To overcome this shortcoming CDF measured a differential cross section for $t \bar{t}$ production as a function of the cosine of the top quark production angle with respect to the proton's direction defined in the $t \bar{t}$ rest frame $\left(\cos \left(\theta_{t}\right)\right)$. To characterize the shape of the cross section CDF employed a projection onto the Legendre polynomials. The Legendre polynomials $P_{l}(x)$ are a complete set of orthogonal polynomials with even powers being symmetric around zero and odd powers - antisymmetric. A detailed analysis of a tree-level $2 \rightarrow 2$ scattering process [15] suggests that the
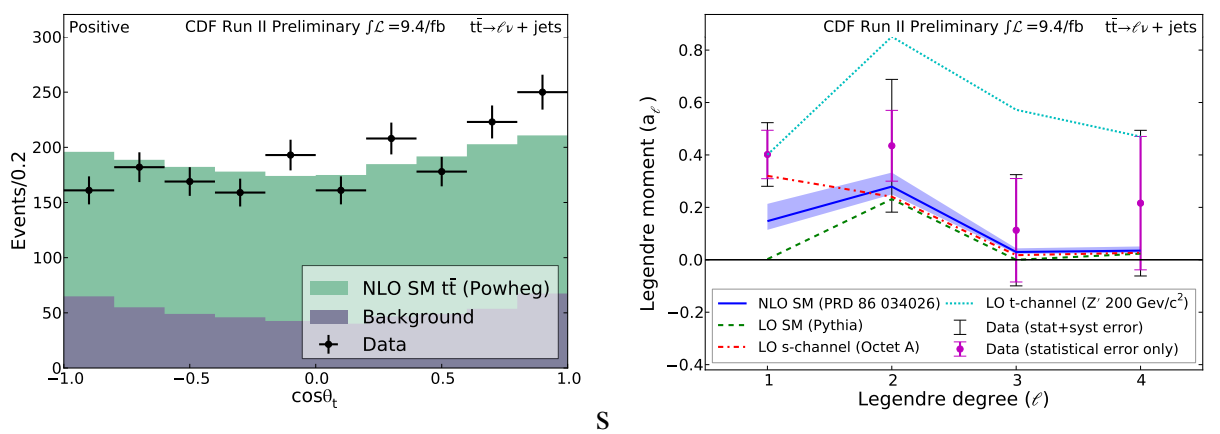

Figure 1: (a) Distribution over $\cos \left(\theta_{t}\right)$ (defined in text) measured by CDF in lepton+jets channel, (b) coefficient in front of $i^{\text {th }}$ Legendre polynomial measured in data.

cross section of a process that is a result of an interference between diagrams that proceed with the total angular momentum $J$ and $J^{\prime}$ is described by a linear combination of the Legendre polynomials of power $l$ such that $\left|J-J^{\prime}\right| \leq l \leq\left|J+J^{\prime}\right|$. In the SM $q \bar{q} \rightarrow t \bar{t}$ production is mediated by spin 1 gluon. Thus, Legendre polynomials of power 0 and 2 can contribute to the production cross section of this process. Polynomial of power 1 is not allowed because it would violate $P$-parity, which is conserved in the strong interactions. Beyond tree-level other powers $l$ are allowed. Thus, a sig- 
nificantly larger than SM-predicted contribution of power 1 polynomial would signify a process mediated by a spin 1 particle that violates $P$-parity.

Using $9.4 \mathrm{fb}^{-1}$ of data CDF selected 3864 events in $l+$ jets channel, out of which $2750 \pm$ 427 are estimated to originate from the $t \bar{t}$ production. The distribution over $\cos \left(\theta_{t}\right)$ is presented in Fig. 1(a). Fig. 1(b) shows the cross section Legendre expansion coefficients in front of the polynomial of power $l$ compared to the theoretical predictions based on SM [16] and some of the new physics models (axigluons and exchange of Z' boson in t-channel). All coefficients are in a good agreement with the SM prediction with a possible exception of the $l=1$ power which exceed the NLO SM prediction by about 1 standard deviation.

\section{Asymmetry in lepton production from $t \bar{t}$ decay}

\subsection{CDF measurement in the $l+$ jets channel}

Forward-backward asymmetry defined using leptons charge $\left(q_{l}\right)$ times its rapidity $\left(y_{l}\right)$ is sensitive not only to asymmetry at production but also to top polarization [17], thus providing an additional discrimination between models. CDF performed an analysis of $9.4 \mathrm{fb}^{-1}$ of data. Muons and electrons are selected with pseudo rapidity $(\eta)$ region of \pm 1.1 . The distribution over lepton signed rapidity is shown in Fig. 2(a) and compared to the POWHEG [18] prediction. Asymmetries observed at the different levels of analysis are presented in Table 1. The first row in this Table 1
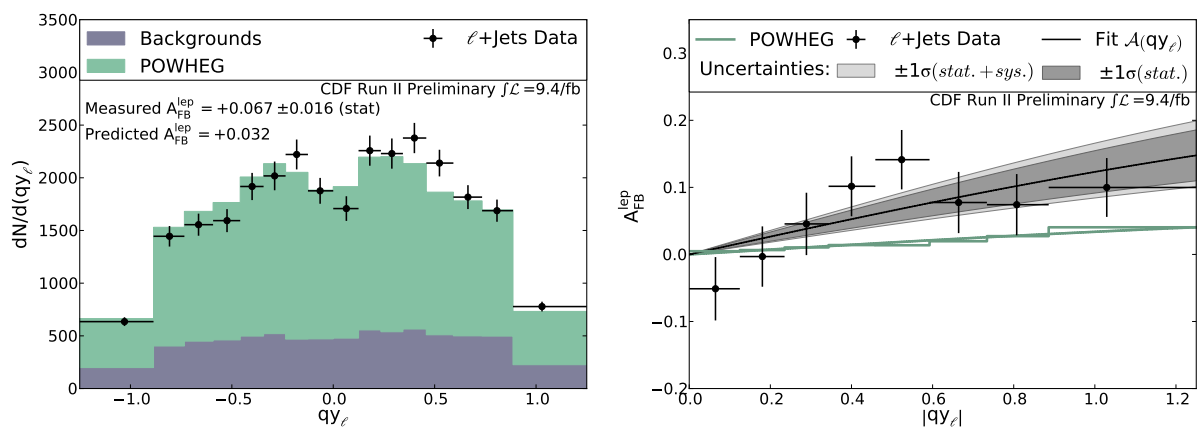

Figure 2: (a) Distribution over $q_{l} y_{l}$ (defined in text) measured by CDF, (b) Dependence of leptonic asymmetry on $\left|q_{l} y_{l}\right|$

\begin{tabular}{|l|c|c|}
\hline Correction level & $A_{\mathrm{FB}}^{l}[\%]$ in CDF data & $A_{\mathrm{FB}}^{l}[\%]$ in POWHEG \\
\hline Raw & $6.7 \pm 1.6$ & 3.2 \\
Background subtracted & $7.0 \pm 1.9 \pm 1.1$ & 2.3 \\
Fully extrapolated & $9.4 \pm 2.4_{-1.7}^{+2.2}$ & 2.7 \\
\hline
\end{tabular}

Table 1: Observed $A_{\mathrm{FB}}^{l}$ for selected $t \bar{t} l+$ jets events compared to POWHEG prediction. The first quoted uncertainty is statistical, the second one is systematic.

represents raw numbers observed in data, while the second row presents the asymmetries corrected for the background contribution. Both these asymmetries corresponds to the events within the fiducial volume, namely for events that have leptons within $|\eta|<1$.1. Assumptions on the behavior 
of the asymmetry must be made to extrapolate the measurement to the full pseudo rapidity region. Examination of several SM and BSM scenarios suggests that asymmetry dependence on $\left|q_{l} y_{l}\right|$ is well described by hyperbolic tangent. Assuming this functional dependence of the asymmetry shown in Fig. 2(b) CDF extrapolates the measurement to the full kinematic region, which is the number presented in the last row of Table 1 . This extrapolation is necessarily model dependent. Once the procedure is applied to several axigluon simulated samples (with mass of $200 \mathrm{GeV}$, width of $50 \mathrm{GeV}$ and assumed left, right and axial couplings to quarks) the extrapolated asymmetry is in a good agreement for left-handed and axial mediators, while it differs by $6 \%$ for right-handed mediators.

\subsection{D0 measurement in the $l+$ jets channel}

D0 also updated its measurement of the $A_{\mathrm{FB}}^{l}$ in $l+$ jets channel and included a study of the asymmetry dependence on the transverse momentum of the lepton $\left(p_{T}^{l}\right)$ from $t \bar{t}$ decay. Since this measurement does not require a full reconstruction of the $t \bar{t}$ system it can be easily extended to $l+3$ jets sample, where one of the jets from $t \bar{t}$ decay is lost. Since the purity differs significantly depending on the number of jets and $b$-tags the $A_{\mathrm{FB}}^{l}$ was measured separately in each subsample and then combined. The distributions in $q_{l} y_{l}$ for four subsamples is presented in Fig. 3. The combined asymmetry at the reconstruction level is $2.8 \pm 2.1(\text { stat })_{-0.9}^{+1.2}($ syst $) \%$ compared to MC @ NLO prediction of $1.6 \%$ [19]. Once corrected for the detector acceptance and resolution the asymmetry is $4.0 \pm 2.3(\text { stat })_{-1.5}^{+1.2}($ syst $) \%$ compared to MC @ NLO prediction of 2.3\%. Contrary to CDF result these numbers correspond to asymmetry within fiducial volume, that is for leptons within rapidity range of 1.5. New D0 result on $A_{\mathrm{FB}}^{l}$ is considerably smaller than previously reported $15.2 \pm 3.8 \%$ measured on $5.4 \mathrm{fb}^{-1}$ of data [12]. Extensive effort went into understanding the source of this change. In addition to updating the result to the full statistics of Run II D0 made several modifications to the analysis strategy to maximize the statistical sensitivity. Most notably these are addition of $l+3$ jets sample, use of a wide range of triggers and improvements to the $b$-tagging algorithm. The strategy was finalized before looking at data. No single modification resulted in a change in the measured asymmetry of more than $2.5 \%$. Moreover, once the current analysis is applied to the $l+4$ jets $1 b$-tag subsample of $5.4 \mathrm{fb}^{-1}$ data the measured asymmetry is in excellent agreement with the published result. At the same time the relative weight of each subsample defined by jet multiplicity and the number of $b$-tags changed to take into the account the expected sensitivity of each subsample, while in the old analysis all subsamples contributed with the equal weight. The conclusion of this investigation is that neither of the measurements is flawed and the change in the measured asymmetry represents a regression to the mean value.

Asymmetry dependence on the lepton kinematics provides an additional information that helps to distinguish between different models. The leading source of background to $t \bar{t}$ signal is production of $W$ boson in association with jets. Leptons from the decay of the inclusively produced $W$ bosons are produced asymmetrically in forward and backward direction due to the higher fraction of the proton momentum carried by $u$ quark compared to that of $d$ quark and due to $V-A$ coupling at $\mathrm{W}$ boson decay. This effect is well established theoretically and experimentally. The simulated asymmetry in $W+$ jets production is an extrapolation from the inclusive one. At the same time, there are notable difference between the inclusive production of $W$ boson and its production in association with jets, for which a quark-gluon initial state plays a more significant role. The fraction 

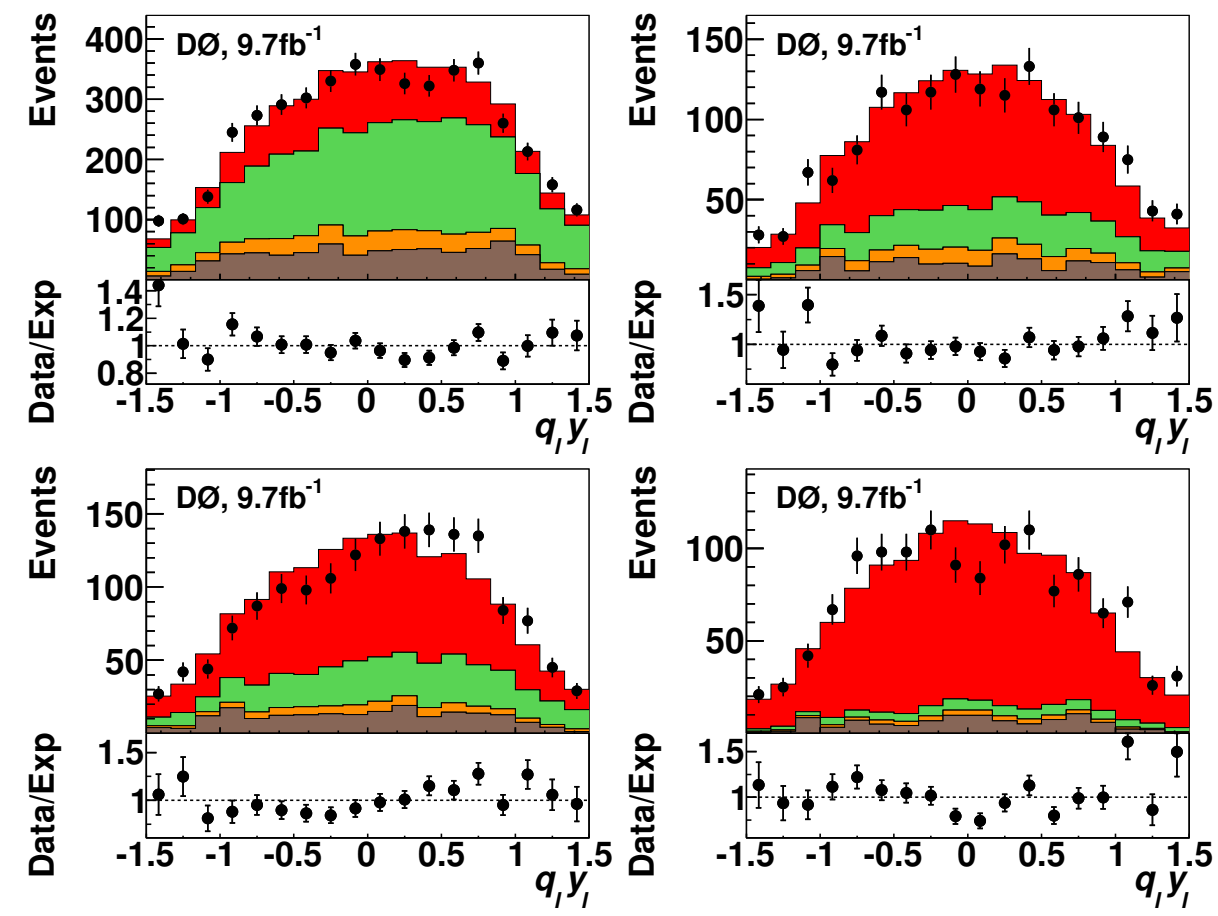

Figure 3: Distribution over lepton's $q_{l} y_{l}$ (defined in text) measured by D0 in lepton+jets channel for events with 3 jets and 1 b-tag(a), 3 jets and 2 or more b-tags(b), 4 or more jets and 1 b-tag(c) and 4 or more jets and 2 or more b-tags $(d)$

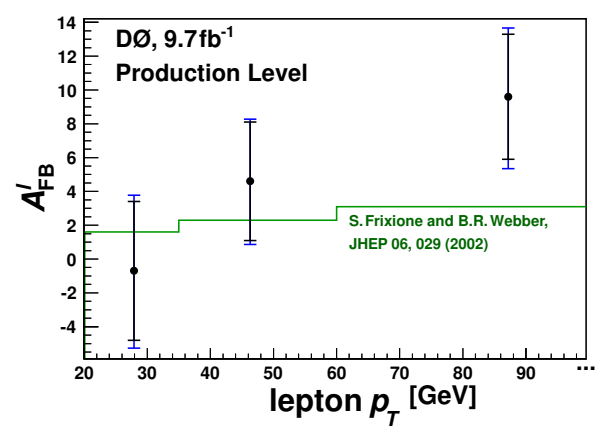

Figure 4: Dependence of $A_{F B}^{l}$ on lepton $p_{T}(\mathrm{~d})$.

of this contribution depends on the $p_{T}^{l}$. To reduce the effect of the extrapolation from the inclusive production D0 used $l+3$ jets sample with zero $b$-tags to calibrate the asymmetry from the $W+$ jets. This subsample is not used for the $t \bar{t}$ asymmetry measurement. The $A_{\mathrm{FB}}^{l}$ measured in data after subtracting the contribution of the calibrated $W+$ jets background as well as other other background sources is shown in Fig. 4 compared to the MC@ NLO prediction.

\subsection{D0 measurement in the dilepton channel}

D0 performed measurements of asymmetry in lepton production in dilepton $t \bar{t}$ events. Two leptons (electrons or muons) are selected to have opposite charge. Two jets are required to be present in events containing two leptons of the same flavor, while at least one jet must be present in $e \mu$ events, where one jets and two or more jet events are treated separately to account for the 
difference in purity. The corresponding distributions in signed pseudo rapidity of leptons (two entries per event) and in the difference in pseudo rapidity between positively and negatively charged leptons are shown in Fig. 5.

The asymmetries based on both quantities are defined for leptons within pseudo rapidity region of $|\eta|<2.0$ that have $|\Delta \eta|<2.4$. The observed asymmetries are corrected for the detector acceptance. To extrapolated from the fiducial region to the full acceptance D0 used the scaling factor derive in MC@ NLO, as a ratio of the full asymmetry to the one defined for the events that contain the leptons within the fiducial region at the generator level. The corrected and extrapolated asymmetries are presented in Table 2. The results are compared to the NLO calculation [16]. The results are largely in agreement with the theoretical prediction.
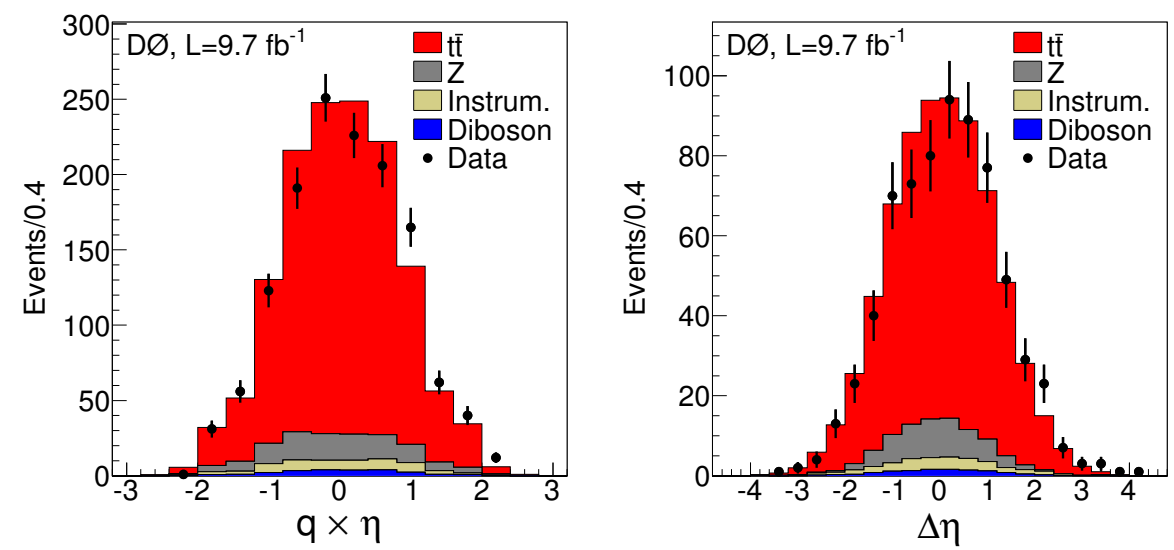

Figure 5: (a) Distribution over lepton's $q \eta$ (defined in text) measured by D0 in dilepton channel, (b) distribution in difference in pseudo-rapidity between two leptons from $t \bar{t}$ decay $(\Delta \eta)$.

\begin{tabular}{|l|c|c|}
\hline$A_{\mathrm{FB}}^{l}[\%]$ & in D0 data & in SM NLO \\
\hline Corrected & $4.1 \pm 3.5 \pm 1.0$ & \\
Extrapolated & $4.4 \pm 3.7 \pm 1.1$ & $3.8 \pm 0.3$ \\
\hline$A_{\mathrm{FB}}^{l l}[\%]$ & & \\
\hline Corrected & $10.5 \pm 4.7 \pm 1.1$ & \\
Extrapolated & $12.3 \pm 5.4 \pm 1.5$ & $4.8 \pm 0.4$ \\
\hline
\end{tabular}

Table 2: Observed $A_{\mathrm{FB}}^{l}$ and $A_{\mathrm{FB}}^{l l}$ for selected $t \bar{t}$ dilepton events compared to SM NLO prediction. The first quoted uncertainty is statistical, the second one is systematic.

\section{References}

[1] S. Abachi et al. (D0 Collaboration), Phys. Rev. Lett. 74, 2632 (1995);

F. Abe et al. (CDF Collaboration), Phys. Rev. Lett. 74, 2626 (1995).

[2] G. Aadet al. (ATLAS Collaboration), Phys. Lett. B 716,1 (2012);

S. Chatrchyanet al. (CMS Collaboration), Phys. Lett. B 716, 30 (2012).

[3] C.T. Hill, Phys. Lett. B 345, 483 (1995). 
[4] P. H. Frampton, J. Shu, and K. Wang, Phys. Lett. B 683, 294 (2010).

[5] A. Djouadi, G. Moreau, F. Richard, and R. K. Singh, Phys. Rev. D 82, 071702 (2010); E. L. Berger et al., Phys. Rev. Lett. 106, 201801 (2011).

[6] L. G. Almeida, G. Sterman, and W. Vogelsang, Phys. Rev. D 78, 014008 (2008); M. T. Bowen, S. D. Ellis, and D. Rainwater, Phys. Rev. D 73, 014008 (2006); S. Dittmaier, P. Uwer, and S. Weinzierl, Phys. Rev. Lett. 98, 262002 (2007); K. Melnikov and M. Schulze, Nucl. Phys. B 840, 129 (2010); J. H. Kuhn and G. Rodrigo, arXiv:1109.6830 [hep-ph] (2011);

[7] A. Falkowski, M. L. Mangano, A. Martin, G. Perez and J. Winter, Phys. Rev. D 87, 034039 (2013).

[8] V. M. Abazov et al. (D0 Collaboration), Phys. Rev. Lett. 100, 142002 (2008).

[9] T. Aaltonen et al. (CDF Collaboration), Phys. Rev. Lett. 101, 202001 (2008).

[10] J. H. Kuhn and G. Rodrigo, Phys. Rev. Lett. 81, 49 (1998).

[11] T. Aaltonen et al. (CDF Collaboration), Phys. Rev. D 83, 112003 (2011).

[12] V. Abazov et al. (D0 Collaboration), Phys. Rev. D 84, 112005 (2011).

[13] W. Hollik and D. Pagani, Phys. Rev. D 84, 093003 (2011).

[14] M. Czakon, A. Mitov, J. High Energy Phys. 1301, 080 (2013).

[15] M. Jacob, G.C. Wick, Ann. Phys. (NY) 7 (4) 404-428 (1959).

[16] W. Bernreuther and Z. -G. Si, Phys. Rev. D 86 (2012) 034026.

[17] E.g., D. Choudhury, R. M. Godbole, S. D. Rindani and P. Saha, Phys. Rev. D 84014023 (2011); E.L. Berger, Q.-H. Cao, C.-R. Chen and H. Zhang, Phys. Rev. D 88, 014033 (2013).

[18] S. Alioli, P. Nason, C. Oleari and E. Re, JHEP 1006 (2010) 043.

[19] S. Frixione and B.R. Webber, JHEP 0206 (2002) 029 ;

S. Frixione et al., JHEP 0308 (2003) 007. 